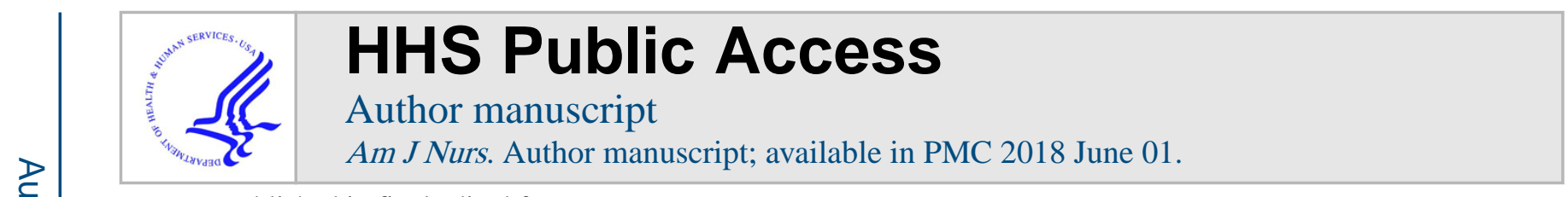

Published in final edited form as:

Am J Nurs. 2017 June ; 117(6): 48-55. doi:10.1097/01.NAJ.0000520253.57459.01.

\title{
Irritable Bowel Syndrome: A review
}

\author{
Kristen Ronn Weaver, MS, \\ National Institute of Nursing Research \\ New York University, Rory Meyers College of Nursing \\ Gail D'Eramo Melkus, EdD, and \\ New York University, Rory Meyers College of Nursing \\ Wendy A Henderson, PhD, MSN, FAAN \\ National Institute of Nursing Research, NIH, DHHS
}

\begin{abstract}
Irritable Bowel Syndrome (IBS) is a common, chronic gastrointestinal condition defined by disturbances in bowel habits and abdominal pain, in the absence of known organic pathology. IBS reduces patients' quality of life, and exerts a substantial, negative impact on financial resources of patients and society at large. The diagnosis of IBS is made according to a symptom based classification system, the Rome Criteria, with updated guidelines, Rome IV, recently released. Physiological and psychological variables have been identified as playing a role in the etiology and perpetuation of symptomatology, highlighting the cross-talk between the brain and the gut in patients living with IBS. Although research investigations have made gains in understanding the pathophysiology of IBS, therapeutic interventions remain symptom driven, with both pharmacological and non-pharmacological approaches in the treatment arsenal. The purpose of this review is to summarize the epidemiology, pathophysiology, diagnosis, and treatment of IBS, and to discuss implications for clinical nursing practice.
\end{abstract}

\section{Keywords}

Irritable Bowel Syndrome; IBS; Rome Criteria; treatment approaches

\section{Introduction}

Irritable bowel syndrome (IBS) is a chronic disorder of the gastrointestinal tract, characterized by abdominal pain and alterations in bowel habits (Canavan, West \& Card, 2014). IBS is the disorder most commonly encountered by gastroenterologists (Halmos, Power, Shepherd, Gibson \& Muir, 2014), and diagnosis is made according to a symptombased classification system, the Rome Criteria, with the latest version, Rome IV, recently released (Drossman, 2016). Prevalence rates in North America have been reported as approximately $12 \%$, and symptoms occur more often in patients less than 50 years of age

Correspondence to: Kristen R. Weaver, MS, ACNP, ANP, HNP, Pre-doctoral Fellow, National Institute of Nursing Research, NIH, DHHS, Digestive Disorders Unit, Division of Intramural Research, 10 Center Drive, Rm 2-2339, Bethesda, MD 20892, UNITED STATES, 301-827-0880, FAX: 301-480-1413, kristen.weaver@nih.gov. 
(Lovell \& Ford, 2012). Patients with IBS suffer not only from gastrointestinal distress, but approximately 40-60\% experience comorbid psychological disorders, such as depression or anxiety (Dekel, Drossman \& Sperber, 2013). In addition, patients with IBS report higher levels of somatization compared to patients without IBS but with gastrointestinal symptoms (Patel et al., 2014). Not surprisingly, IBS has been shown to negatively impact patients' quality of life, as well as to adversely affect society's financial resources.

Despite the substantial cost of IBS to both patients and society, curative, medical interventions have yet to be discovered. The development and persistence of IBS symptoms have been acknowledged as multifactorial in nature, making treatment of the disorder a complicated, clinical endeavor. Approaches are based on the reduction of patient symptomatology, and current pharmacological management often provides suboptimal relief (Grundmann \& Yoon, 2014). Evidence of biological dysregulation has been reported in patients with IBS, and efforts are ongoing to advance understanding of the neurohormonal underpinnings of the disorder (Camilleri, 2014). Although gains have been made in understanding the pathophysiology of IBS, exact mechanisms leading to symptom development are not completely understood (Mayer \& Tillish, 2011). The prevalence of patients who suffer from IBS, developments in the field of IBS research, and the release of updated diagnostic criteria were the impetus for undertaking this review to summarize the epidemiology, pathophysiology, diagnosis, and treatment of IBS, and to discuss implications for clinical nursing practice.

\section{Epidemiology and Burden of Disease}

Incidence rates of IBS are not typically calculated, and prevalence estimates fluctuate internationally, both between and within countries (Canavan, West \& Card, 2014). Such discrepancies have been attributed to the heterogeneity of prevalence investigations, including variations in the use of instruments, methods, diagnostic criteria, populations and cultures assessed (Sperber et al., 2016). This recent investigation evaluated 83 communitybased studies of IBS prevalence from around the world, confirming the global predominance of females who suffer from IBS, but could not confidently estimate a global prevalence rate due to the heterogeneity amongst investigations. The pooled prevalence rate for North America, Europe, Australia and New Zealand was cited as $8.1 \%$ by this investigation (Sperber et al., 2016).

In contrast to the variability of prevalence estimates, the burden or adverse impact of IBS is unfortunately, more consistent. IBS is not associated with increased mortality rates, yet imparts a significant burden to both patients and society as a result of direct medical costs, lost productivity, and reduced health-related quality of life (Inadomi, Fennerty \& Bjorkman, 2003). In 2010, the diagnosis of IBS accounted for over 2 million ambulatory visits in the United States, including visits to the office, emergency departments, and hospital outpatient departments (Peery et al., 2015). Fiscal estimates of lost productivity, lost leisure time, and IBS management exceed one billion dollars in the United States (Everhart \& Ruhl, 2009).

Numerous investigations have established the disproportionate utilization of health care resources by patients with IBS, as well as the negative impact of IBS on patients' quality of 
life (Agarwal \& Spiegel, 2011). Despite the increase in medical attention that patients with IBS receive, many patients experience difficulties and problems with their healthcare encounters (Ringstrom, Sjovall, Simren \& Ung, 2013). Patients with IBS have reported unsupportive encounters with their healthcare providers, shaped by experiences of humiliation, insignificance, and abandonment (Hakanson, Sahlberg-Blom \& Ternestedt, 2010). An additional burden of IBS falls on those who live with or care for people with the disorder; studies report adverse effects on the quality of life of partners of IBS patients (Canavan, West \& Card, 2014). In summary, IBS is a common gastrointestinal condition that has high comorbidity rates, reduces the quality of life of patients, and exerts a substantial, negative impact on the monetary resources of both patients and society.

\section{Pathophysiology}

IBS is recognized as a multifactorial disorder, with the following among the proposed mechanisms contributing to symptomatology: gastrointestinal dysmotility, inflammation, visceral hypersensitivity, and altered intestinal microbiota (Lee \& Park, 2014). Diet and stress exposure (including early life events) have been proposed as contributing factors to this heterogeneous disorder (Heitkemper, Jarrett \& Jun, 2013). Because stress has been identified as a mechanism in the development of IBS, the major components of the stress response system, the autonomic nervous system (ANS) and the hypothalamic-pituitaryadrenal (HPA) axis, have been the subject of numerous investigations of IBS (Fichna \& Storr, 2012). Lastly, genetic predisposition and environmental interactions, such as familial susceptibility and psychosocial stressors, have been implicated in the multifactorial pathogenesis of IBS (Fukudo \& Kanazawa, 2011).

Physiological and psychological variables have jointly been identified as playing a role in the etiology and perpetuation of IBS symptomatology. In an investigation of risk factors for military personnel developing IBS, Riddle et al. (2016) found the risk of developing IBS to be increased after cases of infectious gastroenteritis, and also to be increased in subjects with post-traumatic stress disorder (PTSD), increased number of life stressors, and self-reported anxiety and depression. These findings illustrate the interdependence or cross-talk between the brain and the gut in IBS, a connection commonly known as the brain-gut axis or (BGA).

The BGA refers to pathways among physiological systems, including the central nervous system (CNS), the enteric nervous system (ENS), and the autonomic nervous system (ANS) (Ohman \& Simren, 2007). Endocrine, neural, and neuroimmune pathways facilitate bidirectional communication between the CNS and the gut (Fichna \& Storr, 2012). Patients with IBS have been noted to exhibit disturbances in the BGA, including central and autonomic functions, peripheral factors, peptides, and hormones (Camilleri, 2014). Differences in central processing mechanisms of the BGA have also been demonstrated in IBS patients compared to healthy controls, through the use neuroimaging techniques (Stasi, Rosselli, Bellini, Laffi \& Milani, 2012). Such investigations illustrate that patients with IBS exhibit differences in brain structure, connectivity, and functional responsiveness in comparison to healthy controls (Weaver, Sherwin, Walitt, Melkus \& Henderson, 2016). It is proposed that the clinical presentation of IBS patients with pain, psychological comorbidities, and altered gut motility may be explained through changes in the BGA, 
although mechanisms are not fully understood (Coss-Adame \& Rao, 2014). Despite great progress in understanding the pathophysiology of IBS, such discoveries have not been fully translated to the clinical arena; patient diagnosis remains one primarily of exclusion, and treatment interventions remain symptom driven.

\section{Diagnosis}

IBS is the gastrointestinal disorder that is most commonly diagnosed; the diagnosis is made from the presence of certain symptoms in the absence of organic disease (Chey, Kurlander \& Eswaran, 2015). IBS has been categorized as a functional bowel disorder, defined by symptom onset greater than six months and recurrence at least three days per month during the last three months (Longstreth et al., 2006). Diagnostic criteria require abdominal discomfort or pain to be associated with two or more of the following: improvement with defecation, onset associated with change in the form of stool, or onset associated with a change in the frequency of stool (Longstreth et al., 2006). This diagnostic criteria, known as Rome III classification, also subtypes IBS patients based on their predominant stool pattern: constipation (IBS-C), diarrhea (IBS-D), mixed (IBS-M) or unsubtyped (IBS-U) (Longstreth et al., 2006). Rome III has served as the symptom-based, diagnostic criteria for IBS since its release in 2006; in early 2016 the Rome Foundation released Rome IV, an updated classification system for conceptualizing and diagnosing functional gastrointestinal disorders (Drossman, 2016) (see Table 1).

The Rome IV definition of IBS maintains symptom chronicity (greater than six months) and current activity (present within the prior three months); however, symptom frequency has been changed to at least one day per week (from at least three days per month), specifically requires abdominal pain (discomfort has been eliminated) to be related to (versus improved with) defecation, and the "onset" of abdominal pain has been eliminated from the association of pain with changes in stool (Lacy et al., 2016). Rome IV also updates the subtyping of IBS patients (IBS-C, IBS-D, IBS-M and IBS-U), in that stool type is based on days with abnormal bowel movements, as opposed to bowel movements on all days (Lacy et al., 2016). In addition, Rome IV retains the Bristol Stool Form Scale (Lewis \& Heaton, 1997) as a useful tool to categorize bowel habit (Lacy et al., 2016).

In order to make an accurate diagnosis of IBS, it is recommended to incorporate Rome IV Criteria along with the following: patient history (including dietary questions), physical examination (including anorectal examination), limited laboratory tests (such as complete blood count, C-reactive protein or fecal calprotectin, possible celiac disease serology), and when indicated (either due to recommended guidelines for patient age, alarm signs, family history, etc.), a colonoscopy and/or upper gastrointestinal endoscopy as well as other tests (Lacy et al., 2016). Alternative diagnoses that should be considered when patients present with IBS symptomatology include celiac disease, microscopic colitis (MC), inflammatory bowel disease (including Crohn's disease and ulcerative colitis), bile acid malabsorption, colorectal cancer, and dyssynergic defecation (Chey, Kurlander \& Eswaran, 2015).

The symptoms of IBS and other gastrointestinal diagnoses commonly overlap in clinical practice, therefore, investigations have examined the prevalence of such associations. For 
instance, Halpin \& Ford (2012) conducted a systematic review and meta-analysis on IBS symptoms in patients with inflammatory bowel disease (IBD). These authors report that IBS symptoms were significantly higher in IBD patients with active disease and in remission (overall prevalence approximately 40\%), than in comparison to non-IBD controls. In order to guide therapy for such patients (IBD with IBS-type symptoms), it has been suggested to measure fecal calprotectin as an initial evaluation, and that clinical trials are needed to foster evidence-based approaches (Quigley, 2016). Symptoms also overlap between IBS and another diagnosis, MC, although therapeutic interventions are quite different (Kamp, Kane $\&$ Ford, 2016). This recent systematic review and meta-analysis reported the pooled prevalence of IBS to be approximately $33 \%$ in patients with MC, although these odds were not higher than in other patients with diarrhea. Such overlap of symptoms between patients with IBS and other gastrointestinal disorders, has prompted the use of invasive medical procedures in efforts to obtain a diagnosis. This situation has fostered the efforts of biomarker development to aid in the assessment and diagnosis of patients with IBS.

Over the years, various biomarkers have been proposed to differentiate patients with IBS from healthy controls. For instance, Lembo et al. (2009) proposed a ten biomarker bloodbased panel which included a cytokine, nerve growth factor, autoantibody, antibodies, etc. A few years later, Jones et al. (2014) built on these original ten parameters and compiled an extensive panel (34 markers) that incorporated serological markers, gene expression, and psychological measures. Biomarker initiatives have also investigated colonic transit and fecal bile acids (Camilleri et al., 2014), anti-cytolethal distending toxin B, and anti-vinculin antibodies for distinguishing patients with IBS-D (Pimentel et al., 2015) as well as tests of fecal dysbiosis to characterize patients with IBS (Casen et al., 2015). Although these tests are encouraging in their diagnostic ability and highlight the biological underpinnings of IBS, biomarkers have yet to become the gold standard in clinical practice for diagnosing patients with IBS.

\section{Treatment}

\section{Pharmacological Interventions}

IBS is a multifactorial disorder, and approaches to treatment are similarly diverse. Patient education and reassurance by the provider, however, should be included in all treatments (Halland \& Saito, 2015). Pharmacological management is mainly geared towards the predominant bowel symptom of the patient (diarrhea or constipation), and in patients with IBS-M, a combination of treatments may be needed (Lacy, Chey \& Lembo, 2015). Therapeutic options for relieving abdominal pain in IBS patients include antispasmodics, peppermint oil, selective serotonin reuptake inhibitors (SSRIs), and tricyclic antidepressants; interventions for bowel disturbances (IBS-D) include opioid agonists, antibiotics, bile salt sequestrants, probiotics, mixed opioid agonists/antagonists, and 5-HT3 antagonists, IBS-C is treated with chloride channel activators, polyethylene glycol (PEG), psyllium, and guanylate cyclase C agonists (Lacy et al., 2016) (see Table 2).

The American Gastroenterological Association (AGA) released guidelines on the pharmacological management of IBS, with interventions based on patient subgroups of IBSC and IBS-D (Weinberg, Smalley, Heidelbaugh \& Sultan, 2014). These guidelines vary in 
the level of recommendation from conditional (weak) to strong, and are based on the quality level of the evidence (very low, low, moderate, and high). Included in these guidelines are recommendations for nine pharmaceutical agents in the treatment of IBS: linaclotide, lubiprostone, PEG laxatives, rifaximin, alosetron, loperamide, tricyclic antidepressants, SSRIs and antispasmodics. Overall, the only strong pharmacotherapy recommendation, with high-quality evidence, is the use of linaclotide, a guanylate cyclase $\mathrm{C}$ agonist, for patients with IBS-C.

The American College of Gastroenterology (ACG) performed a recent systematic review on the efficacy of eleven therapies (both pharmacological and non-pharmacological) for treating IBS (Ford et al., 2014). Therapies were evaluated in comparison to placebo or no treatment. In addition, treatments were evaluated in relation to predominant stool pattern and adverse events of treatment. Similar to the AGA guidelines, the ACG recommendations include not only strength of the recommendations (weak versus strong), but also the quality of the evidence upon which the recommendations were based (very low, low, moderate, and high). Non-pharmacological therapies evaluated by the ACG included dietary manipulation, fiber/ psyllium, probiotics/prebiotics, peppermint oil, and psychological therapies including hypnotherapy. Overall, the two therapies that received strong recommendations for use, supported by high and moderate quality of evidence, respectively, were the prosecretory agents, linaclotide and lubiprostone, for the treatment of IBS-C.

\section{Non-pharmacological Interventions}

The goal of treatment interventions is to provide IBS patients with symptomatic relief; often this is attempted through the use of non-pharmacological interventions such as mind-body therapies, diet modification, exercise, and other complementary and alternative approaches (Saha, 2014). Conclusions from a Cochrane Review on the efficacy of psychological interventions in IBS patients, were that cognitive behavioral therapy and interpersonal psychotherapy may be beneficial in patients with IBS, although issues were noted regarding study heterogeneity, validity and sample size (Zijdenbos et al., 2009). More recently, Ford et al. (2014) carried out a systematic review and meta-analysis of 30 studies on the effect of psychological therapies in patients with IBS. The authors found beneficial treatment effects of cognitive behavioral therapy, multicomponent psychological therapy, dynamic psychotherapy, and hypnotherapy.

A growing focus of clinical research has been to improve IBS symptomatology through dietary modifications (Chey, 2016). Food ingestion can contribute to the generation of symptoms through stimulation of chemoreceptors, mechanoreceptors, by osmotic actions, altered secretion, activation of motor reflexes, and colonic fermentation (Eswaran, Tack \& Chey, 2011). Patients with IBS are more likely than the general population to report adverse reactions to food; dietary intolerance of IBS patients has been attributed to gluten, wheat, lactose, fermentable oligosaccharides, disaccharides, monosaccharides and polyols (or FODMAPs) as well as fructose malabsorption (Mullin et al., 2014). Exclusion diets have been used in patients with IBS, for instance with wheat and dairy, although conflicting results have been reported (Halland \& Saito 2015). A recent investigation of patients with IBS-D, found a six-week gluten-free diet significantly improved scores of symptom severity, 
anxiety, depression, fatigue, and quality of life (Aziz et al., 2016). The low-FODMAP diet has also been examined in patients with IBS and found to result in improved abdominal and bowel symptoms in some patients, although guidance from a nutritionist is generally recommended (Lacy, Chey \& Lembo, 2015). Another dietary manipulation, the use of soluble fiber supplementation, was found to have beneficial effects on global symptomatology in patients with IBS, according to a systematic review and meta-analysis of 14 investigations (Moayyedi et al., 2014).

Parallel to the focus on dietary modifications are efforts to manipulate or alter the intestinal microbiota. Undigested food is used by intestinal microbes (GI microbiota) upon entering the intestine, and microbiota play a major role in gastrointestinal processes and overall health (Barbara et al., 2016). Therefore, numerous studies have investigated the utility of probiotics in IBS, in efforts to manipulate the intestinal microbiota and improve symptomatology. For instance, a meta-analysis found a positive effect of probiotics in treating IBS pain, abdominal distension and flatulence, but noted further research was needed on variables such as probiotic dose, species, and duration (Ortiz-Lucas et al., 2013). Another systematic review and meta-analysis reported similar findings, concluding that probiotics are effective in treating IBS in terms of global symptoms, flatulence, abdominal pain and bloating, although conclusions could not be drawn regarding individual strains or species. (Ford et al., 2014). Further manipulation of the intestinal microbiota includes initiatives on fecal microbiota transplantation (FMT). Whereas probiotics aim to alter the native gut microbiota, FMT introduces a community of gut micro-organisms to replace or repair the native gut microbiota (Borody \& Khoruts, 2012). Although it is suggested that restoring intestinal homeostasis via FMT might improve symptoms in patients with IBS, this has been performed on a limited number of cases; RCTs are needed to confirm safety and efficacy of the treatment, and the Federal Drug Administration requires an investigational drug application for use (Pinn, Aroniadis \& Brandt, 2015).

Additional types of non-pharmacological interventions have been investigated in IBS patients. For instance, Johannesson et al. (2011) in a randomized control trial of 102 patients, found a 12-week intervention of moderately increased physical activity to decrease symptom severity of IBS and to improve some aspects of quality of life (related to physical role and physical functioning). In addition, this study found that the proportion of IBS symptoms worsening during the study was greater in the physically inactive group than in the physically active one. In a follow-up study (median time 5.2 years), 39 of the prior participants were found to report a positive, long-term effect of moderately increased physical activity on IBS symptomatology, quality of life, depression, anxiety, and fatigue (Johannesson et al., 2015). Yoga is another mind-body intervention that has been explored in the IBS patient population. A recent systematic review, surveying six randomized controlled trials, reports benefits of yoga on gastrointestinal symptoms, anxiety, and quality of life in patients with IBS (Schumann et al., 2016). This investigation could not make recommendations regarding yoga practice for IBS, however, because of the methodological heterogeneity that existed between reviewed studies.

Treatment modalities explored in the IBS patient population have also come from traditional Chinese medicine; these include acupuncture, electroacupuncture (EA) and moxibustion. 
Acupuncture involves the insertion of needles to target acupoints, whereas moxibustion is the application of heat (by ignited moxa) to the acupuncture points (Ma et al., 2014). In an investigation of acupuncture in IBS, Chu et al. (2012) used functional magnetic resonance imaging (fMRI) to evaluate brain activation in patients with IBS-D. These researchers used actual EA and sham EA to evaluate central processing in response to rectal distension, and report significant fMRI differences between acupuncture groups. These authors postulate that acupuncture's therapeutic mechanism may potentially be modulated via the medial and pulvinar nucleus of the thalamus, as well as the right insula. Another fMRI study of patients with IBS-D undergoing rectal distension, evaluated brain imaging changes before and after, sham and actual, moxibustion treatment (Zhu et al., 2014). These researchers report the group who received actual moxibustion experienced decreased IBS symptoms, improved quality of life, and decreased rectal sensitivity; brain regions implicated in these changes include the prefrontal cortex and anterior cingulate cortex. A systematic review and metaanalysis on the efficacy of acupuncture in treating IBS, found patients who received acupuncture to report greater improvement than those receiving pharmacological therapies, although no differences were found between actual and sham acupuncture on IBS symptoms or quality of life (Manheimer et al., 2012).

Investigations have also been performed on non-pharmacological, self-management interventions to diminish IBS symptomatology. Jarrett et al. (2009) evaluated the efficacy of a 9-session, nurse-delivered, comprehensive self-management (CSM) intervention that incorporated cognitive-behavioral strategies, diet, relaxation, and education. This study found significant improvement in GI symptoms and quality of life measures in patients with IBS who underwent CSM intervention in comparison to patients who received usual care (UC). Further analysis of the CSM patients revealed long term, beneficial effects of the intervention, reflected by lower daily levels of patient-reported stress (Deechakawan et al., 2013). Patients with IBS who received the CSM intervention were also found to have significantly greater reductions in daily depression and anxiety scores across three, six and twelve months follow-up, compared with patients with IBS who received UC (Deechakawan et al., 2014). A recent evaluation of this program, found 94\% of CSM participants still incorporating intervention strategies, one year after their last session (Zia et al., 2016). These investigations highlight the potential for nursing-led initiatives to impart long-lasting, positive health effects on patients with IBS. Nurses are in a prime position, as members of the healthcare team, to facilitate improved outcomes for patients living with IBS.

\section{Implications for Clinical Nursing Practice}

Nurses can positively affect the clinical outcomes of patients with IBS in countless ways. Familiarity with prevalence rates of IBS, as well as gender and age predominance, will promote early consideration of IBS as a potential diagnosis. Recognizing disorders that could mimic the signs and symptoms of IBS, as well as understanding Rome IV guidelines, will enable nurses to facilitate an early diagnosis, possibly reducing unnecessary tests or invasive procedures. Once the diagnosis of IBS is made, nursing interventions can truly aid in the well-being of this patient population. Awareness of the advances in the field of IBS research, will enable greater appreciation of the multifactorial nature of the disorder in terms of etiology and perpetuation of symptomatology. Knowledge pertaining to the high rates of 
comorbid psychological disorders, and possible life stressors in this patient population, will allow nurses to tailor communication and patient interaction, accordingly.

Perhaps the mechanism whereby nurses can have the greatest, positive impact upon patients with IBS is through education and support. Nurses who are familiar with pharmacological options for treating the global symptoms of IBS, as well as symptoms by IBS subtype, can actively engage patients regarding medication types and mechanisms of action. By remaining up-to-date on evidence-based reviews of non-pharmacological options, nurses can help patients navigate treatment alternatives to select appropriate interventions. Given the numerous influences upon generation and perpetuation of IBS symptoms, it is important for nurses to question patients about dietary and medication changes, life stressors and support networks, as part of their ongoing assessment. Lastly, nurses should provide reassurance throughout the treatment process, thereby maintaining a therapeutic relationship as efforts are made towards diminishing IBS symptoms, and improving patients' quality of life.

Although IBS is a common, multifactorial, gastrointestinal disorder that exacts a significant toll on both patients and society, significant advances have been made in the field, and therapeutic options show promise. Efforts to understand the many components of IBS, and to recognize the individuality of each IBS patient, are leading the way towards a comprehensive, personalized approach to patient care. Nursing professionals are key players in the collaborative process of patient care, and can aid in the development of diagnostic and therapeutic approaches, both in the clinical and research domain. Such innovation, while remaining attuned to IBS patients' diverse needs, will potentiate therapeutic gains and bring about symptomatic relief, to this deserving patient population.

\section{Acknowledgments}

The authors would like to acknowledge funding from the US Department of Health and Human Services, National Institutes of Health, National Institute of Nursing Research, Division of Intramural Research (KRW: Intramural Research Training Award, Graduate Partnership Program; WAH: 1ZIANR000018, 01-05). Additional support provided to KRW by New York University College of Nursing, Jonas Center for Nursing and Veterans Healthcare, and the Columbia University Presbyterian Hospital School of Nursing Alumni Association.

\section{References}

Agarwal N, Spiegel BM. The effect of irritable bowel syndrome on health-related quality of life and health care expenditures. Gastroenterology Clinics of North America. 2011; 40:11-19. DOI: 10.1016/j.gtc.2010.12.013 [PubMed: 21333898]

Aziz I, Trott N, Briggs R, North JR, Hadjivassiliou M, Sanders DS. Efficacy of a gluten-free diet in subjects with irritable bowel syndrome-diarrhea unaware of their HLA-DQ2/8 genotype. Clinical Gastroenterology and Hepatology. 2016; 14:696-703. DOI: 10.1016/j.cgh.2015.12.031 [PubMed: 26748221]

Barbara G, Feinle-Bisset C, Ghoshal UC, Santos J, Vanner SJ, Vergnolle N, ... Quigley EM. The intestinal microenvironment and functional gastrointestinal disorders. Gastroenterology. 2016; 150:1305-1318. DOI: 10.1053/j.gastro.2016.02.028

Borody TJ, Khoruts A. Fecal microbiota transplantation and emerging applications. Nature Reviews Gastroenterology and Hepatology. 2012; 9:88-96. DOI: 10.1038/nrgastro.2011.244

Camilleri M. Physiological underpinnings of irritable bowel syndrome: Neurohormonal mechanisms. Journal of Physiology. 2014; 592:2967-2980. DOI: 10.1113/jphysiol.2014.270892 [PubMed: 24665101] 
Camilleri M, Shin A, Busciglio I, Carlson P, Acosta A, Bharucha AE, ... Zinsmeister AR. Validating biomarkers of treatable mechanisms in irritable bowel syndrome. Neurogastroenterology and Motility. 2014; 26:1677-1685. DOI: 10.1111/nmo.12421 [PubMed: 25244349]

Canavan C, West J, Card T. The epidemiology of irritable bowel syndrome. Clinical Epidemiology. 2014; 6:71-80. DOI: 10.2147/CLEP.S40245 [PubMed: 24523597]

Canavan C, West J, Card T. Review article: The economic impact of the irritable bowel syndrome. Alimentary Pharmacology \& Therapeutics. 2014; 40:1023-1034. DOI: 10.1111/apt.12938 [PubMed: 25199904]

Casen C, Vebo HC, Sekelja M, Hegge FT, Karlsson MK, Ciemniejewska E, ... Rudi K. Deviations in human gut microbiota: A novel diagnostic test for determining dysbiosis in patients with IBS or IBD. Alimentary Pharmacology \& Therapeutics. 2015; 42:71-83. DOI: 10.1111/apt.13236 [PubMed: 25973666]

Chey WD. Food: The main course to wellness and illness in patients with irritable bowel syndrome. American Journal of Gastroenterology. 2016; 111:366-371. DOI: 10.1038/ajg.2016.12 [PubMed: 26856749]

Chey WD, Kurlander J, Eswaran S. Irritable bowel syndrome a clinical review. Journal of the American Medical Association. 2015; 313:949-958. DOI: 10.1001/jama.2015.0954 [PubMed: 25734736]

Chu WC, Wu JC, Yew DT, Zhang L, Shi L, Yeung DK, ... Sung JJ. Does acupuncture therapy alter activation of neural pathway for pain perception in irritable bowel syndrome?: A comparative study of true and sham acupuncture using functional magnetic resonance imaging. Journal of Neurogastroenterology and Motility. 2012; 18:305-316. DOI: 10.5056/jnm.2012.18.3.305 [PubMed: 22837879]

Coss-Adame E, Rao SS. Brain and gut interactions in irritable bowel syndrome: New paradigms and new understandings. Current Gastroenterology Reports. 2014; 16:379.doi: 10.1007/ s11894-014-0379-z [PubMed: 24595616]

Deechakawan W, Cain KC, Jarrett ME, Burr RL, Heitkemper MM. Effect of self-management intervention on cortisol and daily stress levels in irritable bowel syndrome. Biological Research for Nursing. 2013; 15:26-36. DOI: 10.1177/1099800411414047 [PubMed: 21765120]

Deechakawan W, Heitkemper MM, Cain KC, Burr RL, Jarrett ME. Anxiety, depression, and catecholamine levels after self-management intervention in irritable bowel syndrome. Gastroenterology Nursing. 2014; 37:24-32. DOI: 10.1097/SGA.0000000000000017 [PubMed: 24476829]

Dekel R, Drossman DA, Sperber AD. The use of psychotropic drugs in irritable bowel syndrome. Expert Opinion on Investigational Drugs. 2013; 22:329-339. DOI: 10.1517/13543784.2013.761205 [PubMed: 23316916]

Drossman DA. Functional gastrointestinal disorders: History, pathophysiology, clinical features and Rome IV. Gastroenterology. 2016; 150:1262-1279. DOI: 10.1053/j.gastro.2016.02.032

Eswaran S, Tack J, Chey WD. Food: The forgotten factor in the irritable bowel syndrome. Gastroenterology Clinics of North America. 2011; 40:141-162. DOI: 10.1016/j.gtc.2010.12.012 [PubMed: 21333905]

Everhart JE, Ruhl CE. Burden of digestive diseases in the United States part I: Overall and upper gastrointestinal diseases. Gastroenterology. 2009; 136:376-386. DOI: 10.1053/j.gastro. 2008.12.015 [PubMed: 19124023]

Fichna J, Storr MA. Brain-gut interactions in IBS. Frontiers in Pharmacology. 2012; 3:1-12. doi:UNSP12710.3389/fphar.2012.00127. [PubMed: 22291651]

Ford AC, Moayyedi P, Lacy BE, Lembo AJ, Saito YA, Schiller LR, ... Quigley EM. American College of Gastroenterology monograph on the management of irritable bowel syndrome and chronic idiopathic constipation. American Journal of Gastroenterology. 2014; 109:S2-26. DOI: 10.1038/ ajg.2014.187 [PubMed: 25091148]

Ford AC, Quigley EM, Lacy BE, Lembo AJ, Saito YA, Schiller LR, ... Moayyedi P. Effect of antidepressants and psychological therapies, including hypnotherapy, in irritable bowel syndrome: Systematic review and meta-analysis. American Journal of Gastroenterology. 2014; 109:1350 1365. DOI: 10.1038/ajg.2014.148 [PubMed: 24935275] 
Ford AC, Quigley EM, Lacy BE, Lembo AJ, Saito YA, Schiller LR, ... Moayyedi P. Efficacy of prebiotics, probiotics, and synbiotics in irritable bowel syndrome and chronic idiopathic constipation: Systematic review and meta-analysis. American Journal of Gastroenterology. 2014; 109:1547-1561. DOI: 10.1038/ajg.2014.202 [PubMed: 25070051]

Fukudo S, Kanazawa M. Gene, environment, and brain-gut interactions in irritable bowel syndrome. Journal of Gastroenterology and Hepatology. 2011; 26:110-115. [PubMed: 21443722]

Grundmann O, Yoon SL. Complementary and alternative medicines in irritable bowel syndrome: An integrative view. World Journal of Gastroenterology. 2014; 20:346-362. DOI: 10.3748/ wjg.v20.i2.346 [PubMed: 24574705]

Hakanson C, Sahlberg-Blom E, Ternestedt BM. Being in the patient position: Experiences of health care among people with irritable bowel syndrome. Qualitative Health Research. 2010; 20:11161127. DOI: 10.1177/1049732310369914 [PubMed: 20463359]

Halland M, Saito YA. Irritable bowel syndrome: New and emerging treatments. BMJ. 2015; 350:h1622.doi: 10.1136/bmj.h1622 [PubMed: 26088265]

Halmos EP, Power VA, Shepherd SJ, Gibson PR, Muir JG. A diet low in FODMAPs reduces symptoms of irritable bowel syndrome. Gastroenterology. 2014; 146:67-75. [PubMed: 24076059]

Halpin SJ, Ford AC. Prevalence of symptoms meeting criteria for irritable bowel syndrome in inflammatory bowel disease: Systematic review and meta-analysis. American Journal of Gastroenterology. 2012; 107:1474-1482. DOI: 10.1038/ajg.2012.260 [PubMed: 22929759]

Heitkemper M, Jarrett M, Jun SE. Update on irritable bowel syndrome program of research. Journal of Korean Academy of Nursing. 2013; 43:579-586. DOI: 10.4040/jkan.2013.43.5.579 [PubMed: 24351989]

Inadomi JM, Fennerty MB, Bjorkman D. Systematic review: The economic impact of irritable bowel syndrome. Alimentary Pharmacology \& Therapeutics. 2003; 18:671-682. [PubMed: 14510740]

Jarrett ME, Cain KC, Burr RL, Hertig VL, Rosen SN, Heitkemper MM. Comprehensive selfmanagement for irritable bowel syndrome: Randomized trial of in-person vs. combined in-person and telephone sessions. American Journal of Gastroenterology. 2009; 104:3004-3014. DOI: 10.1038/ajg.2009.479 [PubMed: 19690523]

Johannesson E, Ringstrom G, Abrahamsson H, Sadik R. Intervention to increase physical activity in irritable bowel syndrome shows long-term positive effects. World Journal of Gastroenterology. 2015; 21:600-608. DOI: 10.3748/wjg.v21.i2.600 [PubMed: 25593485]

Johannesson E, Simren M, Strid H, Bajor A, Sadik R. Physical activity improves symptoms in irritable bowel syndrome: A randomized controlled trial. American Journal of Gastroenterology. 2011; 106:915-922. DOI: 10.1038/ajg.2010.480 [PubMed: 21206488]

Jones MP, Chey WD, Singh S, Gong H, Shringarpure R, Hoe N, ... Talley NJ. A biomarker panel and psychological morbidity differentiates the irritable bowel syndrome from health and provides novel pathophysiological leads. Alimentary Pharmacology \& Therapeutics. 2014; 39:426-437. DOI: 10.1111/apt.12608 [PubMed: 24387672]

Kamp EJ, Kane JS, Ford AC. Irritable bowel syndrome and microscopic colitis: A systematic review and meta-analysis. Clinical Gastroenterology and Hepatology. 2016; 14:659-668. DOI: 10.1016/ j.cgh.2015.09.031 [PubMed: 26453949]

Lacy BE, Chey WD, Lembo AJ. New and emerging treatment options for irritable bowel syndrome. Gastroenterology \& Hepatology. 2015; 11:1-19.

Lacy BE, Mearin F, Chang L, Chey WD, Lembo AJ, Simren M, Spiller R. Bowel disorders. Gastroenterology. 2016; 150:1393-1407. DOI: 10.1053/j.gastro.2016.02.031

Lee YJ, Park KS. Irritable bowel syndrome: Emerging paradigm in pathophysiology. World Journal of Gastroenterology. 2014; 20:2456-2469. DOI: 10.3748/wjg.v20.i10.2456 [PubMed: 24627583]

Lembo AJ, Neri B, Tolley J, Barken D, Carroll S, Pan H. Use of serum biomarkers in a diagnostic test for irritable bowel syndrome. Alimentary Pharmacology \& Therapeutics. 2009; 29:834-842. DOI: 10.1111/j.1365-2036.2009.03975.x [PubMed: 19226291]

Lewis SJ, Heaton KW. Stool form scale as a useful guide to intestinal transit time. Scandinavian Journal of Gastroenterology. 1997; 32:920-924. DOI: 10.3109/00365529709011203 [PubMed: 9299672] 
Longstreth GF, Thompson WG, Chey WD, Houghton LA, Mearin F, Spiller RC. Functional bowel disorders. Gastroenterology. 2006; 130:1480-1491. DOI: 10.1053/j.gastro.2005.11.061 [PubMed: 16678561]

Lovell RM, Ford AC. Global prevalence of and risk factors for irritable bowel syndrome: A metaanalysis. Clinical Gastroenterology and Hepatology. 2012; 10:712-721. DOI: 10.1016/j.cgh. 2012.02.029 [PubMed: 22426087]

Ma XP, Hong J, An CP, Zhang D, Huang Y, Wu HG, ... Meeuwsen S. Acupuncture-moxibustion in treating irritable bowel syndrome: How does it work? World Journal of Gastroenterology. 2014; 20:6044-6054. DOI: 10.3748/wjg.v20.i20.6044 [PubMed: 24876727]

Manheimer E, Wieland LS, Cheng K, Li SM, Shen X, Berman BM, Lao L. Acupuncture for irritable bowel syndrome: Systematic review and meta-analysis. American Journal of Gastroenterology. 2012; 107:835-847. DOI: 10.1038/ajg.2012.66 [PubMed: 22488079]

Mayer EA, Tillisch K. The brain-gut axis in abdominal pain syndromes. Annual Review of Medicine. 2011; 62:381-396. DOI: 10.1146/annurev-med-012309-103958

Moayyedi P, Quigley EM, Lacy BE, Lembo AJ, Saito YA, Schiller LR, ... Ford AC. The effect of fiber supplementation on irritable bowel syndrome: A systematic review and meta-analysis. American Journal of Gastroenterology. 2014; 109:1367-1374. DOI: 10.1038/ajg.2014.195 [PubMed: 25070054]

Mullin GE, Shepherd SJ, Chander Roland B, Ireton-Jones C, Matarese LE. Irritable bowel syndrome: Contemporary nutrition management strategies. Journal of Parenteral and Enteral Nutrition. 2014; 38:781-799. DOI: 10.1177/0148607114545329 [PubMed: 25085503]

Ohman L, Simren M. New insights into the pathogenesis and pathophysiology of irritable bowel syndrome. Digestive and Liver Disease. 2007; 39:201-215. DOI: 10.1016/j.dld.2006.10.014 [PubMed: 17267314]

Ortiz-Lucas M, Tobias A, Saz P, Sebastian JJ. Effect of probiotic species on irritable bowel syndrome symptoms: A bring up to date meta-analysis. Revista Espanola de Enfermedades Digestivas. 2013; 105:19-36. [PubMed: 23548007]

Patel P, Bercik P, Morgan DG, Bolino C, Pintos-Sanchez MI, Moayyedi P, Ford AC. Irritable bowel syndrome is significantly associated with somatisation in 840 patients, which may drive bloating. Alimentary Pharmacology \& Therapeutics. 2015; 41:449-458. DOI: 10.1111/apt.13074 [PubMed: 25586008]

Peery AF, Crockett SD, Barritt AS, Dellon ES, Eluri S, Gangarosa LM, ... Sandler RS. Burden of gastrointestinal, liver, and pancreatic diseases in the United States. Gastroenterology. 2015; 149:1731-1741. e1733. DOI: 10.1053/j.gastro.2015.08.045 [PubMed: 26327134]

Pimentel M, Morales W, Rezaie A, Marsh E, Lembo A, Mirocha J, ... Chang C. Development and validation of a biomarker for diarrhea-predominant irritable bowel syndrome in human subjects. Plos One. 2015; 10:1-12. DOI: 10.1371/journal.pone.0126438

Pinn DM, Aroniadis OC, Brandt LJ. Is fecal microbiota transplantation (FMT) an effective treatment for patients with functional gastrointestinal disorders (FGID)? Neurogastroenterology and Motility. 2015; 27:19-29. DOI: 10.1111/nmo.12479 [PubMed: 25424663]

Quigley EM. Overlapping irritable bowel syndrome and inflammatory bowel disease: Less to this than meets the eye? Therapeutic Advances in Gastroenterology. 2016; 9:199-212. DOI: 10.1177/1756283X15621230 [PubMed: 26929782]

Riddle MS, Welsh M, Porter CK, Nieh C, Boyko EJ, Gackstetter G, Hooper TI. The epidemiology of irritable bowel syndrome in the US military: Findings from the millennium cohort study. American Journal of Gastroenterology. 2016; 111:93-104. DOI: 10.1038/ajg.2015.386 [PubMed: 26729548]

Ringstrom G, Sjovall H, Simren M, Ung EJ. The importance of a person-centered approach in diagnostic workups of patients with irritable bowel syndrome: A qualitative study. Gastroenterology Nursing. 2013; 36:443-451. DOI: 10.1097/SGA.0000000000000011 [PubMed: 24304529]

Saha L. Irritable bowel syndrome: Pathogenesis, diagnosis, treatment, and evidence-based medicine. World Journal of Gastroenterology. 2014; 20:6759-6773. DOI: 10.3748/wjg.v20.i22.6759 [PubMed: 24944467] 
Schumann D, Anheyer D, Lauche R, Dobos G, Langhorst J, Cramer H. Effect of yoga in the therapy of irritable bowel syndrome: A systematic review. Clinical Gastroenterology and Hepatology. 2016; doi: 10.1016/j.cgh.2016.04.026

Sperber AD, Dumitrascu D, Fukudo S, Gerson C, Ghoshal UC, Gwee KA, ... Whitehead W. The global prevalence of IBS in adults remains elusive due to the heterogeneity of studies: A Rome Foundation working team literature review. Gut. 2016; 0:1-8. DOI: 10.1136/gutjnl-2015-311240

Stasi C, Rosselli M, Bellini M, Laffi G, Milani S. Altered neuro-endocrine-immune pathways in the irritable bowel syndrome: The top-down and the bottom-up model. Journal of Gastroenterology. 2012; 47:1177-1185. DOI: 10.1007/s00535-012-0627-7 [PubMed: 22766747]

Weaver KR, Sherwin LB, Walitt B, Melkus GD, Henderson WA. Neuroimaging the brain-gut axis in patients with irritable bowel syndrome. World Journal of Gastrointestinal Pharmacology and Therapeutics. 2016; 7:320-333. DOI: 10.4292/wjgpt.v7.i2.320 [PubMed: 27158548]

Weinberg DS, Smalley W, Heidelbaugh JJ, Sultan S. American Gastroenterological Association Institute guideline on the pharmacological management of irritable bowel syndrome. Gastroenterology. 2014; 147:1146-1148. DOI: 10.1053/j.gastro.2014.09.001 [PubMed: 25224526]

Zhu Y, Wu Z, Ma X, Liu H, Bao C, Yang L, ... Wu H. Brain regions involved in moxibustion-induced analgesia in irritable bowel syndrome with diarrhea: A functional magnetic resonance imaging study. BMC Complementary and Alternative Medicine. 2014; 14:500.doi: 10.1186/1472-6882-14-500 [PubMed: 25516481]

Zia JK, Barney P, Cain KC, Jarrett ME, Heitkemper MM. A comprehensive self-management irritable bowel syndrome program produces sustainable changes in behavior after 1 year. Clinical Gastroenterology and Hepatology. 2016; 14:212-219. DOI: 10.1016/j.cgh.2015.09.027 [PubMed: 26453951]

Zijdenbos IL, de Wit NJ, van der Heijden GJ, Rubin G, Quartero AO. Psychological treatments for the management of irritable bowel syndrome. Cochrane Database of Systematic Reviews. 2009; 1:CD006442.doi: 10.1002/14651858.CD006442.pub2 


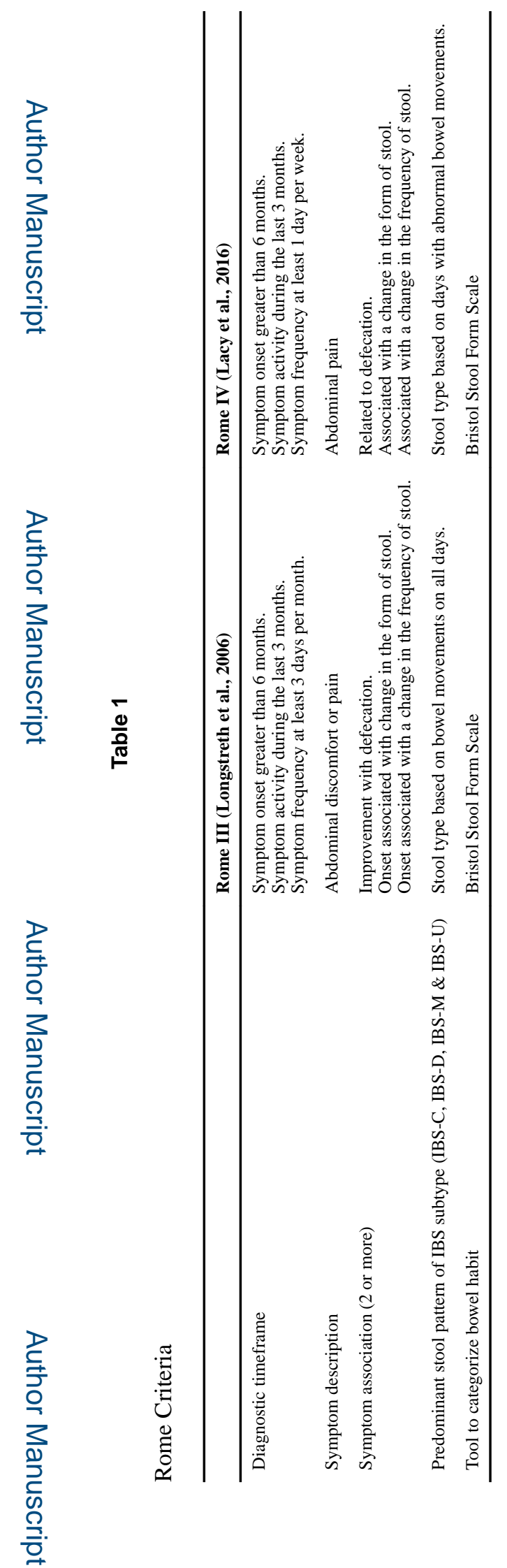

Am J Nurs. Author manuscript; available in PMC 2018 June 01. 


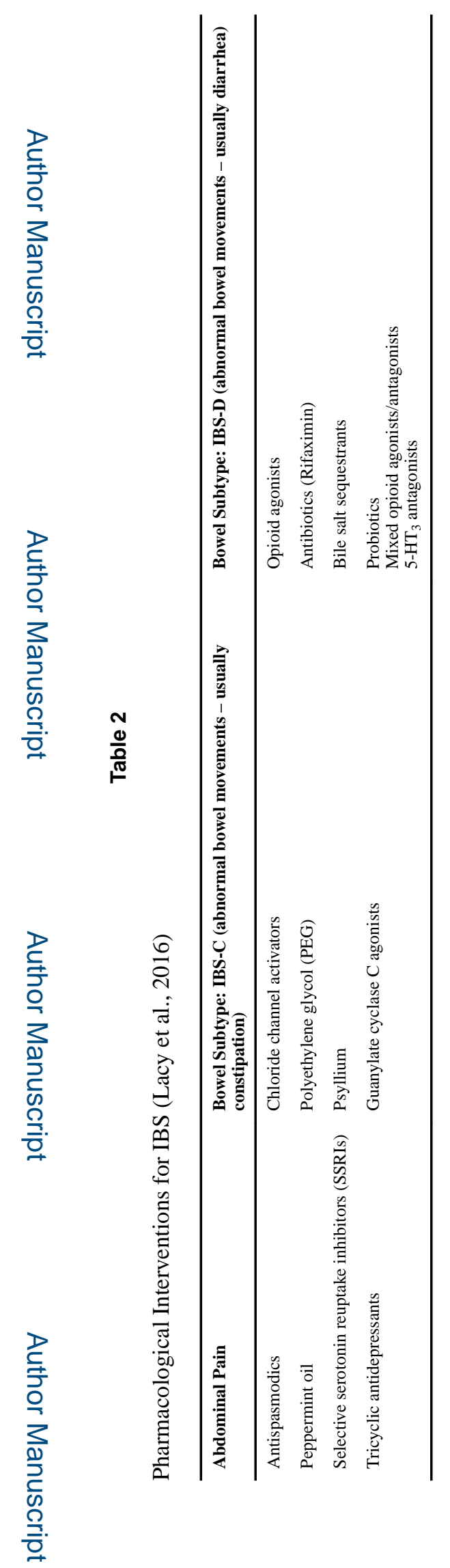

Am J Nurs. Author manuscript; available in PMC 2018 June 01. 